\title{
Rapid induction of IgE responses to a worm cysteine protease during murine pre-patent schistosome infection
}

\author{
Lucia A de Oliveira Fraga ${ }^{1,2}$, Erika W Lamb ${ }^{1,5}$, Elizabeth C Moreno ${ }^{3}$, Mitali Chatterjee ${ }^{1}$, Jan Dvořák4, \\ Melaine Delcroix ${ }^{4,6}$, Mohammed Sajid ${ }^{4,7}$, Conor R Caffrey ${ }^{4}$, Stephen J Davies ${ }^{1 *}$
}

\begin{abstract}
Background: During the pre-patent stage of infection, juvenile Schistosoma blood flukes co-opt signals from the adaptive immune system to facilitate parasite development, but the types of responses that are induced at this early stage of infection, and the parasite antigens they target, have not been characterized.

Results: Through analysis of experimental pre-patent infections, we show that the S. mansoni cysteine protease SmCB1 is rapidly targeted by an antigen-specific lgE response. The induction of this response is independent of schistosome eggs as infection with male or female worms alone also induced SmCB1-specific lgE. We also show that the SmCB1-specific lgE response is dependent on cognate CD4 ${ }^{+} \mathrm{T}$ cell help and IL-4, suggesting that prepatent Th2 responses provide T cell help for the SmCB1-specific IgE response. Finally, exposed human subjects also produced lgE against SmCB1.

Conclusions: Our data demonstrate that, like eggs, schistosome worms also induce functional type 2 responses and that a parasite cysteine protease is an inducer of type 2 responses during the early stages of schistosome infection.
\end{abstract}

\section{Background}

Despite their large size and complex multicellular structure, schistosomes display a remarkable ability to survive for years within the mammalian bloodstream, remaining viable and reproductively active in the face of potentially damaging immune responses. Mechanisms proposed to account for the ability of schistosomes to evade immune destruction include, for example, molecular "camouflage", achieved by adsorption of host molecules to the parasite surface; molecular "mimicry", through expressing antigens with amino acid sequences that are similar or identical to host proteins; continuous surface membrane turn-over; and modulation of immune responses so that potentially harmful effector mechanisms are downregulated or inhibited [1].

While schistosomes mostly evade immune injury during natural infection, acquired immunity to schistosome

\footnotetext{
* Correspondence: sdavies@usuhs.mil

'Department of Microbiology and Immunology, Uniformed Services

University of the Health Sciences, Bethesda, MD 20814, USA

Full list of author information is available at the end of the article
}

worms that interferes with infection can be demonstrated under some circumstances, both in naturally exposed human subjects [2] and laboratory animal models of vaccine-induced immunity [3]. Although the precise mechanisms by which protection is mediated under these different circumstances are debated [2], there is consensus that protective immunity is dependent on $\mathrm{CD}^{+}{ }^{+} \mathrm{T}$ cell responses [2]. Intriguingly, there is also evidence that Schistosoma blood flukes exploit $\mathrm{CD}^{+}{ }^{+} \mathrm{T}$ cell responses, by co-opting the activities of $\mathrm{CD} 4^{+} \mathrm{T}$ cells during pre-patent infection to promote parasite development and subsequent reproduction $[4,5]$. The mechanisms by which $\mathrm{CD} 4^{+} \mathrm{T}$ cells facilitate schistosome development have yet to be fully elucidated, but these findings suggest that extensive co-evolution has resulted in a host-parasite relationship where schistosomes induce $\mathrm{CD} 4^{+} \mathrm{T}$ cell responses that are conducive to establishment of infection, while simultaneously avoiding immune injury. An understanding of the $\mathrm{CD}_{4}^{+} \mathrm{T}$ cell responses induced by schistosome worms during pre-patent infection is therefore a prerequisite to 
elucidating how these parasites evade immune injury and establish productive infections.

Unlike the response to schistosome eggs [6], the $\mathrm{CD} 4^{+}$ $\mathrm{T}$ cell responses induced by schistosome worms, especially during normal permissive infection, have not been extensively characterized. Schistosome eggs are potent inducers of Th2 responses [7], and some of the major immunodominant antigens of eggs have been identified [8-10]. Indeed, an egg-secreted ribonuclease, omega-1, was recently identified as the principle component of eggs that conditions dendritic cells for Th2 polarization $[11,12]$. In contrast, the $\mathrm{CD}^{+} \mathrm{T}$ cell response to schistosome worms during the pre-patent phase of infection has been characterized as a Th1 response [13]. Recently we demonstrated that pre-patent schistosome infection and infections with either male or female worms alone that preclude the possibility of egg production, also induce type 2 responses, characterized by induction of $\mathrm{CD} 4^{+} \mathrm{T}$ cells and basophils that produce IL- 4 in response to worm antigens [14]. Thus the immune response to developing schistosome worms during primary infection is more complex than previously appreciated and there is likely much still to learn about the immunological context within which primary schistosome infection is established. For example, the worm antigens that are the main targets of pre-patent responses have yet to be described. Specific worm antigens have been identified in the context of immune resistance, such as in vaccinated animals [15-17] and putatively resistant human subjects [18-20], but the significance of these antigens during normal permissive infection has not been explored.

In this study, we attempted to identify worm antigens that stimulate $\mathrm{CD} 4^{+} \mathrm{T}$ cell responses during permissive primary infection, as these antigens may be involved in stimulating responses that facilitate schistosome worm development. Because $\mathrm{CD} 4^{+} \mathrm{T}$ cell responses to individual antigens are difficult to detect directly in mice, owing to the low frequency of $\mathrm{CD} 4^{+} \mathrm{T}$ cells with specificity for any single antigen [21], we used isotype classswitching of antibody responses as a marker for $\mathrm{CD} 4^{+} \mathrm{T}$ cell responses, since antibody isotype-switching by $\mathrm{B}$ cells requires cognate $\mathrm{CD} 4^{+} \mathrm{T}$ cell help [22]. Our results reveal that the parasite gut-associated $S$. mansoni cysteine protease cathepsin B1 (SmCB1; Sm31) [23] is an immunodominant target of adaptive responses during pre-patent infection, demonstrating that the pre-patent response to schistosome worms is focused and specific, and is not simply characterized by immunosuppression or nonspecific polyclonal responses. Further analysis of the prepatent response demonstrated the rapid establishment of an antigen-specific IgE response to SmCB1, which was dependent on T cell help and IL-4 but independent of schistosome eggs. Analysis of human subjects who reside in endemic areas suggests that $\mathrm{SmCB} 1$ is also the target of IgE responses in humans. Together our data suggest that, like schistosome eggs, schistosome worms also induce type 2 responses and that a worm cysteine protease is involved in type 2 response induction.

\section{Results}

Immunodominant worm antigens targeted by adaptive responses during pre-patent infection

To identify the earliest evidence of class-switching in the humoral response to worm antigens during pre-patent infection, ELISAs using plate-bound soluble worm antigen (SWAP) were used to detect SWAP-specific IgM, IgG1 and IgG2b in plasma of infected mice at $0,1,2,3,4$ and 8 weeks post infection (Figure $1 \mathrm{~A}-\mathrm{C}$ ). The first significant production of non-IgM antibodies with specificity for worm antigens was detected at 4 weeks post infection, when SWAP-specific IgG1 was detected (Figure 1B). To identify the worm antigens targeted by this IgG1 response during primary schistosome infection, a SWAP preparation was probed by immunoblot, using plasma from mice that were infected for $2,3,4$ or 5 weeks. Bound antibodies were detected with enzymeconjugated secondary antibodies specific for murine IgM, IgG1, and IgG2b and total mouse IgG. Results obtained with anti-IgG1 are presented in Figure 1. Similar results were obtained with anti-IgM and pan-specific anti-IgG secondary antibodies (data not shown). Using anti-IgG1, no reactivity was detected at 2 weeks (Figure 1D) and 3 weeks (Figure 1E) post infection, but intense reactivity with an apparent single species of approximately $31 \mathrm{kDa}$ molecular mass was evident by 4 weeks post infection (Figure 1F). In concordance with this result, the presence of worm antigen-specific IgG1 was also first detected at 4 weeks post infection using a whole worm antigen-based ELISA (Figure 1B). By 5 weeks post infection (Figure 1G), weak reactivity with additional species of approximately 42 and $64 \mathrm{kDa}$ was also evident, in addition to the 31 $\mathrm{kDa}$ species. Thus, the humoral response during a primary, pre-patent schistosome infection predominantly targets a worm antigen or antigens of approximately 31 $\mathrm{kDa}$. Furthermore, the production of antigen-specific IgG suggests this antigen is also the target of a $\mathrm{CD} 4^{+} \mathrm{T}$ helper response, because isotype class-switching by $\mathrm{B}$ cells to production of isotypes other than IgM requires cognate $\mathrm{CD} 4^{+} \mathrm{T}$ cell help, in the form of cytokines and CD40CD40L interactions [22].

\section{SmCB1 is the target of an IgE response during pre-patent schistosome infection}

Previous studies have shown that the $S$. mansoni gut protease cathepsin B1 (SmCB1; also known as Sm31), the mature form of which has a molecular mass of approximately $31 \mathrm{kDa}$, is highly antigenic during schistosome 

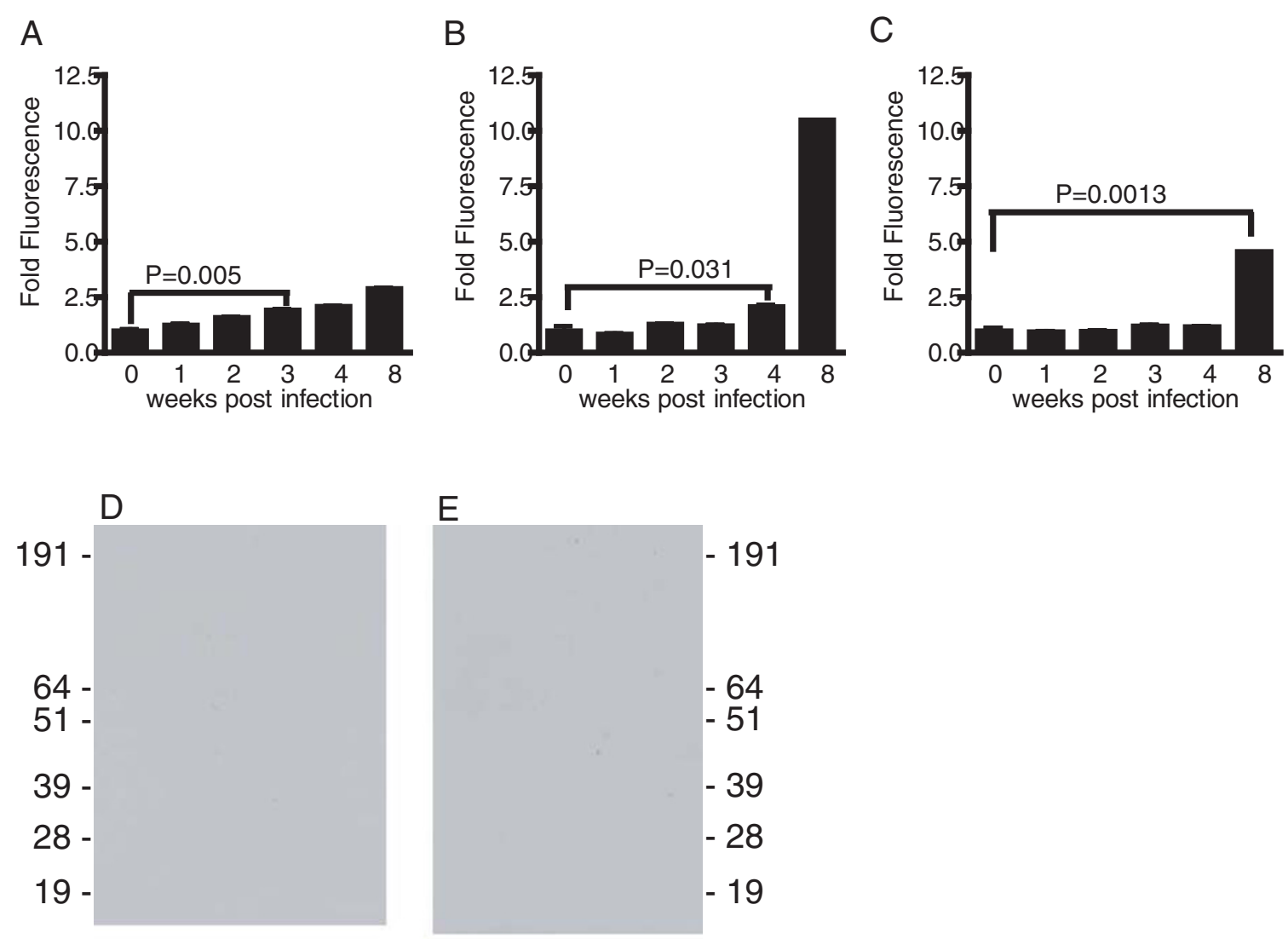

$\mathrm{E}$
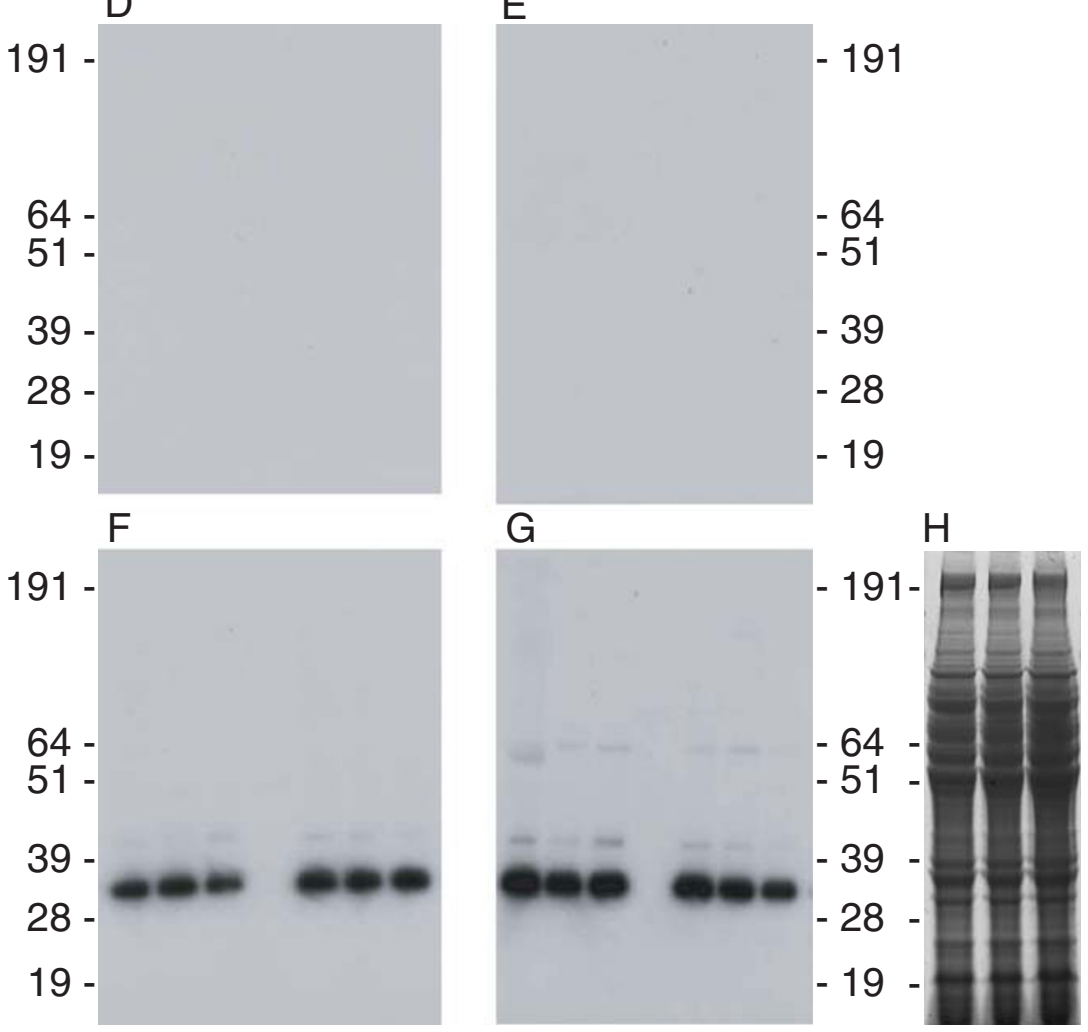

Figure 1 lgG1 elicited during pre-patent schistosome infection is primarily specific for antigens of $\mathbf{3 1} \mathbf{k D a}$. Worm antigen-specific lgM (A) IgG1 (B) and lgG2b (C) in the plasma of wild type mice with pre-patent (2,3 and 4 weeks post infection) and patent infection (8 weeks post infection) were quantified by ELISA. Data are displayed as relative fluorescence units (RFU). P values for the first time points to show significant increases in antibody level are shown (obtained by Dunn's post-test following Kruskal-Wallis test). Experimental groups consisted of five mice per group. Data are representative of three independent experiments. Triplicate samples of two different SWAP preparations were separated by SDS-PAGE and probed by immunoblotting with plasma from infected mice at 2 (D), 3 (E), 4 (F) and 5 (G) weeks post infection. Bound lgG1 was detected with an alkaline phosphatase-conjugated goat anti-mouse lgG1 antibody. H, SDS-PAGE of three SWAP samples, stained for total protein by Coomassie Blue staining. Positions of protein molecular weight markers are indicated in $\mathrm{kDa}$. Data are representative of three independent experiments.

infection, both in human patients [24-26] and in experimentally infected mice [27]. Thus we hypothesized that the $31 \mathrm{kDa}$ species recognized by the pre-patent response shown in Figure 1 corresponded to SmCB1. To test this hypothesis, we tested plasma samples from infected mice for the presence of SmCB1-specific antibodies by ELISA, using recombinant, non-glycosylated SmCB1 as antigen. Using this more sensitive technique, SmCB1-specific IgM was detectable in plasma as early as 2 weeks post infection (Figure 2A). Isotype class-switching of the anti-SmCB1 response became apparent at 3 weeks post infection, when SmCB1-specific IgG1 antibodies were first detected in 

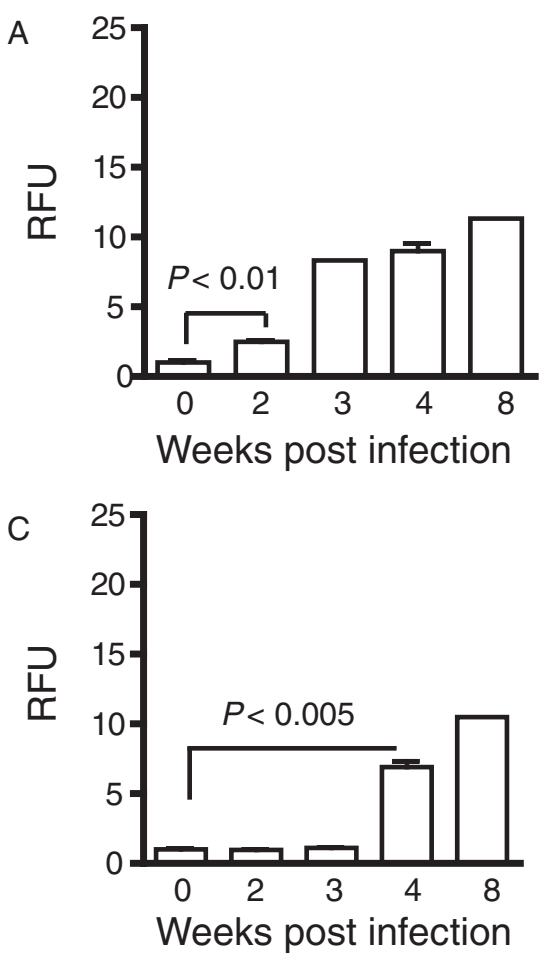

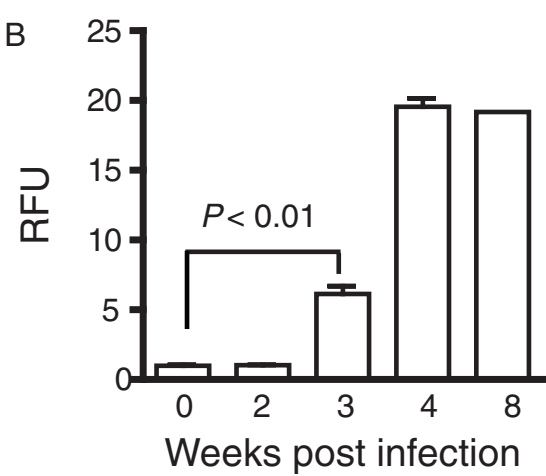

D

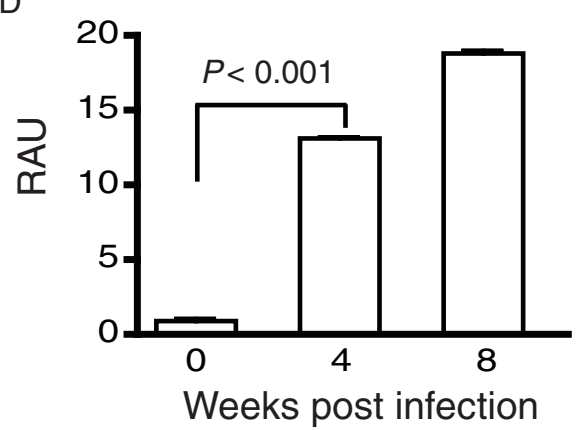

Figure 2 Relative concentrations of SmCB1-specific immunoglobulin isotypes in plasma of mice with pre-patent schistosome infection SmCB1-specific IgM (A), IgG1 (B), IgG2b (C) and IgE (D) in the plasma of wild type mice with pre-patent (2, 3 and 4 weeks post infection) and patent infection (8 weeks post infection) were quantified by ELISA. Data are displayed as relative fluorescence units (RFU) for lgM (A), IgG1 (B) and IgG2b (C), and as relative absorbance units (RAU) for lgE (D). P values for the first time points to show significant increases in antibody level are shown (obtained by Dunn's post-test following Kruskal-Wallis test). Experimental groups consisted of five mice per group. Data are representative of three independent experiments.

plasma (Figure 2B). By 4 weeks post infection, SmCB1-specific IgG2b (Figure 2C) and, unexpectedly, IgE (Figure 2D) antibodies were also detectable. These data indicate that the $\mathrm{SmCB} 1$ protein is a target of humoral responses during pre-patent schistosome infection. Furthermore, detection of class-switching in the response to the SmCB1 protein suggests that a $C D 4^{+} \mathrm{T}$ helper response to $\mathrm{SmCB} 1$ is mounted in parallel with the antibody response, as isotype switching requires cognate $T$ cell help [22]. Finally, the detection of SmCB1-specific IgG1 (Figure 2B) and especially IgE (Figure 2D) suggests that SmCB1-specific CD4 ${ }^{+}$ $\mathrm{T}$ cells produce IL-4, as B cell class-switching to production of these isotypes requires cognate $\mathrm{T}$ cell help from IL4-producing $\mathrm{CD}^{+} \mathrm{T}$ cells [28].

\section{Rapid induction of SmCB1-specific IgE in the absence of schistosome eggs}

While previous reports suggested that $C D 4^{+} \mathrm{T}$ cells primarily mount Th1 responses to worm antigens during pre-patent infection $[7,13]$, we recently showed that schistosome worms also induce IL-4-producing $\mathrm{CD}^{+} \mathrm{T}$ cells during pre-patent infection, which could therefore serve as a source of Th2 help for the IgE response demonstrated in Figure 2. Alternatively, Th2 polarization of the $\mathrm{CD} 4^{+} \mathrm{T}$ cell response to SmCB1 could be the result of unexpectedly early oviposition, as schistosome eggs and egg antigens are potent, autonomous inducers of Th2 responses that could bias concomitant responses to worm antigens. To test this latter hypothesis, we examined IgE responses in mice infected with only male or female worms, thus precluding any possibility that the animals were exposed to eggs. Infection with either male or female worms alone both induced significant increases in total plasma IgE concentrations by 4 weeks post infection, with male-only infections inducing more IgE than female worms (Figure 3A). Furthermore, male-only and female-only infections also induced SmCB1-specific IgE responses by 4 weeks post infection, with males again inducing more IgE than females (Figure 3B). Thus, exposure to either male or female worms was sufficient for rapid induction of total and antigen-specific IgE. The results also demonstrate that total and SmCB1-specific IgE responses are not induced as a result of early oviposition. That male worms induced more total and SmCB1-specific IgE than did female 

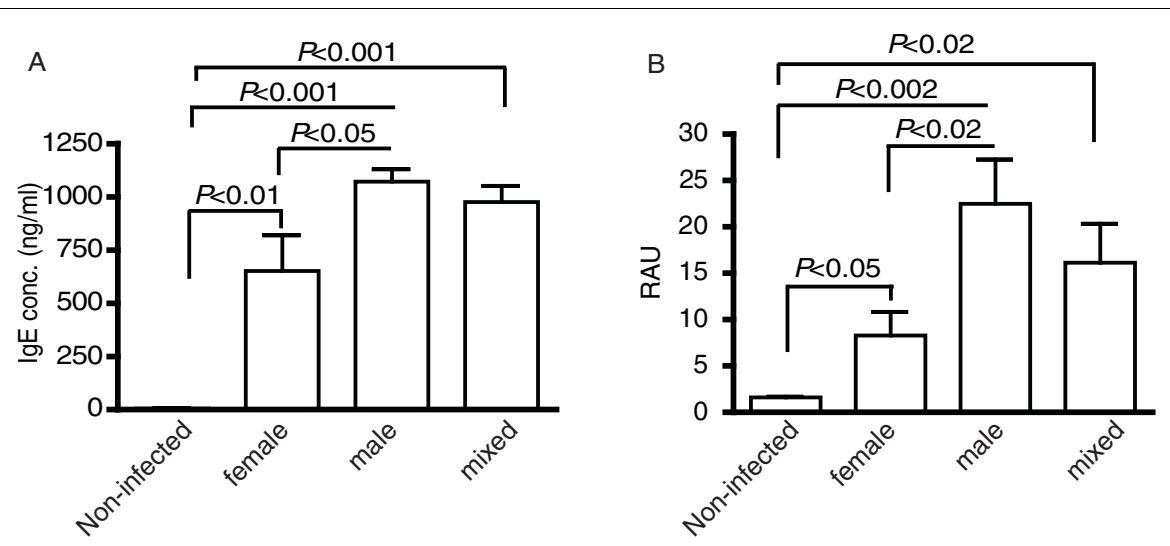

Figure $3 \mathrm{lgE}$ responses to worm antigens are independent of schistosome eggs. Concentrations of total (A) and SmCB1-specific IgE (B) in plasma of mice infected for 4 weeks with female, male or mixed sex infections were determined by ELISA. P values were calculated by Dunn's post-test following Kruskal-Wallis test. Experimental groups consisted of five mice per group. Data are representative of two independent experiments. RAU, relative absorbance units.

worms may be due to the abnormal development of female worms in the absence of males [29].

\section{T cell help and IL-4 are required for SmCB1-specific IgE production during pre-patent infection}

To test whether worm-induced pre-patent Th2 responses, such as the those we have described recently [14], are involved in class-switching of the anti-SmCB1 response to IgE, we tested whether the SmCB1-specific IgE response was dependent on $\mathrm{CD} 4^{+}$ $\mathrm{T}$ cell help and IL-4. First, we examined worm-induced IgE responses in mice where provision of $\mathrm{CD}^{+} \mathrm{T}$ cell help to B cells is prevented through disruption of MHC II gene expression. Consistent with a role for $\mathrm{CD}^{+} \mathrm{T}$ cell help in the SmCB1-specific IgE response, SmCB1-specific IgE antibodies were not detected in the plasma of infected $\mathrm{MHC} \mathrm{II}^{-1-}$ mice (Figure 4A), despite the fact these animals express constitutively high levels of nonspecific natural IgE [14,30]. Second, to test the role of IL-4 in the generation of SmCB1specific IgE, IL-4 activity was blocked in vivo by administration of a neutralizing anti-IL-4 monoclonal antibody. Neutralization of IL-4 completely ablated the production of both total (Figure 4B) and SmCB1-specific IgE (Figure 4C) at 4 weeks post infection, demonstrating that these responses require IL-4. In contrast levels of SmCB1-specific IgG1 and IgG2b were either not affected or augmented by anti-IL- 4 treatment (Figure 4D), demonstrating that this treatment did not result in a general impairment of B cell responses. Together, these data suggest that the SmCB1-specific IgE response during pre-patent infection is dependent on a concomitant IL-4-producing $\mathrm{CD}_{4}^{+} \mathrm{T}$ helper response to worm antigens.
Natural human exposure to schistosome worms induces IgE responses to $\mathrm{SmCB} 1$

As SmCB1 appears to be a potent inducer of antigenspecific IgE responses in infected mice before the onset of egg production, we questioned whether similar responses were induced in humans exposed to schistosome infection. Because human cases of acute schistosome infection, prior to the onset of oviposition, are rarely detected, we chose instead to analyze a cohort of egg-negative or putatively resistant (also known as "endemic normal") Brazilian subjects, who are exposed to schistosome worm antigens but presumably do not experience high levels of egg antigens, as they never show evidence of active, patent infection, i.e. parasite eggs are not detectable in the stool and egg-induced pathology does not develop [2]. Sera from endemic normal subjects contained IgE antibodies specific for SWAP and SmCB1 (Figure 5A), suggesting that, as in mice, human exposure to schistosome worms is sufficient to induce antigen-specific IgE, in the absence of substantial exposure to eggs. Indeed, endemic normal patients exhibited significantly higher levels of SmCB1-specific IgE than did susceptible subjects who experience patent schistosome infections (Figure 5A). In contrast, patients with a history of patent infection exhibited higher levels of SWAP-specific IgG4 than did endemic normal subjects, consistent with the association of IgG4 antibodies with chronic patent schistosomiasis [31].

\section{Discussion}

The haploid genomes of the Schistosoma contain upwards of $3.6 \times 10^{8}$ base pairs and are predicted to encode for 12,000 or more genes [32]. While not all these genes are expressed during the intramammalian 


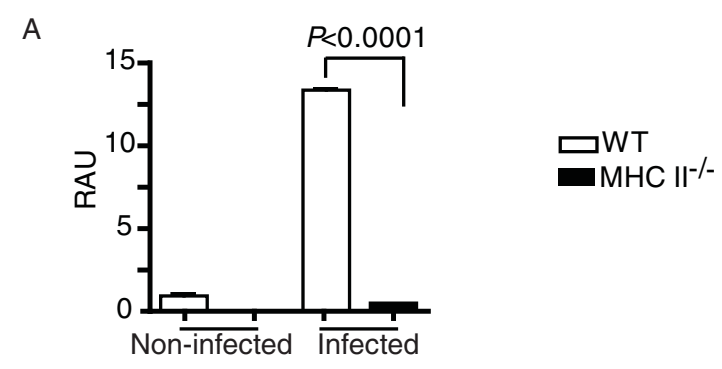

B

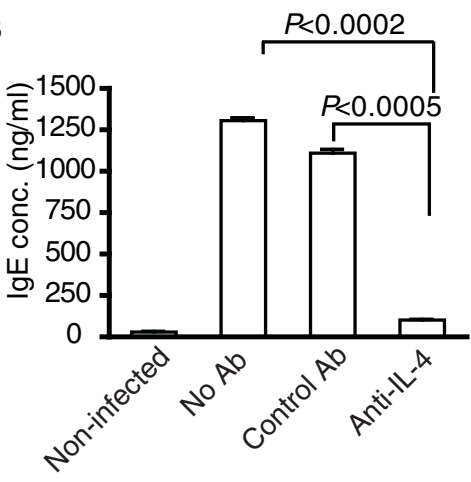

C

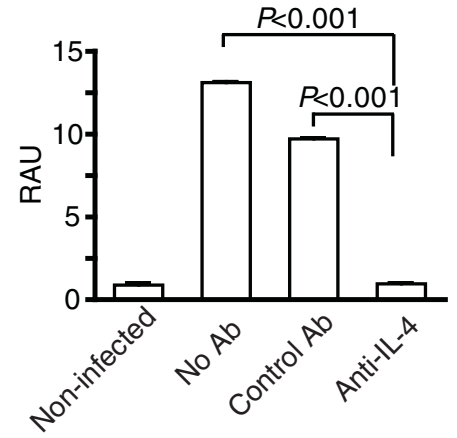

D

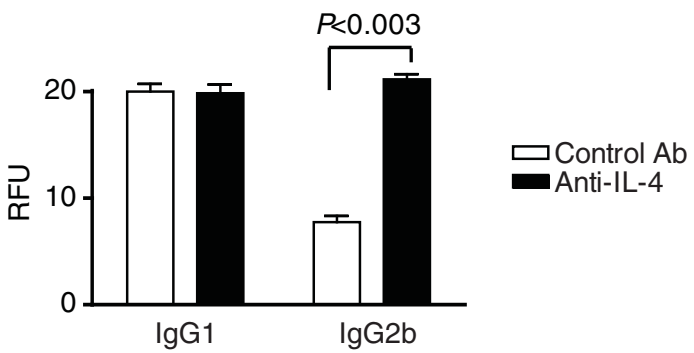

Figure $4 \mathrm{lgE}$ responses to schistosome worms require cognate Th2 help. A, Concentrations of SmCB1-specific lgE in the plasma of wild type and $\mathrm{MHC} \mathrm{I}^{-/-}$mice were measured by ELISA at 4 weeks post infection. Concentrations of total (B) and SmCB1-specific IgE (C) in the plasma of wild type mice that were treated with a neutralizing anti-IL-4 antibody were quantified by ELISA at 4 weeks post infection. D, Concentrations of SmCB1-specific IgG1 and IgG2b in the plasma of wild type mice were treated with neutralizing antiIL-4 antibody were quantified by ELISA at 4 weeks post infection. P values were calculated by Dunn's post-test following Kruskal-Wallis test. Experimental groups consisted of five mice per group. Data are representative of two independent experiments. RAU, relative absorbance units; RFU, relative fluorescence units. phase of the life cycle [33], it is nonetheless unexpected that such an apparently limited number of gene products are recognized by the host immune system after 4 weeks of exposure to the developing worms. While other protein species are clearly targeted by the humoral response during pre-patent infection [34], (Figure 1G), our data suggest that the majority of the antigen-specific antibodies produced during the first five weeks of infection target an antigen of approximately $31 \mathrm{kDa}$. While we cannot exclude the possibility that other protein species are responsible for some of the reactivity in the $31 \mathrm{kDa}$ band, the data in Figure 2 indicate that SmCB1, a known immunogenic protein of $31 \mathrm{kDa}$ molecular mass (in the mature form, [24,35]), is the target of a robust humoral response during the first four weeks of infection. Furthermore, the appearance of SmCB1-specific antibodies at two weeks post infection and the subsequent amplification and class-switching of the response over the following weeks correlates with the known temporal expression pattern of SmCB1. Performing a central nutritive function in the degradation of host proteins within the parasite gut [23], SmCB1 expression is detectable in cercariae [36] and newly transformed schistosomula [37] and is maintained into adulthood [38,39].

While it is tempting to speculate that immune evasion mechanisms employed by the parasite to minimize immune recognition, such as molecular camouflage and mimicry, are factors in limiting the number of antigens to which responses are detected, other mechanisms may contribute to focus the adaptive response on SmCB1. First, the schistosome regularly regurgitates its gut contents, including SmCB1, making the protein available for interaction with the immune system. Second, the potentially inflammatory remains of regurgitated host cells [40] may act as an adjuvant for the comingled SmCB1 when it is encountered by antigen-presenting cells. Finally, recent findings have suggested that cysteine protease activities present immunostimulatory molecular motifs, akin to toll-like receptor ligands such as lipopolysaccharide, but which preferentially initiate Th2 responses rather than Th1 responses [41]. Because vertebrates do not secrete cysteine proteases [42], it is hypothesized that the vertebrate immune system has evolved sensors for the presence of cysteine proteases in extracellular spaces and interprets their presence as a "danger signal". This mechanism may explain the Th2 priming properties of helminths and allergens, as significant cysteine protease activity is frequently associated with these two classes of immunostimulatory agents [43].

In other pathogens, focused responses to immunodominant antigens contribute to pathogen persistence and transmission. For example, in viruses capable of rapid genetic change, such as retroviruses [44], 
A

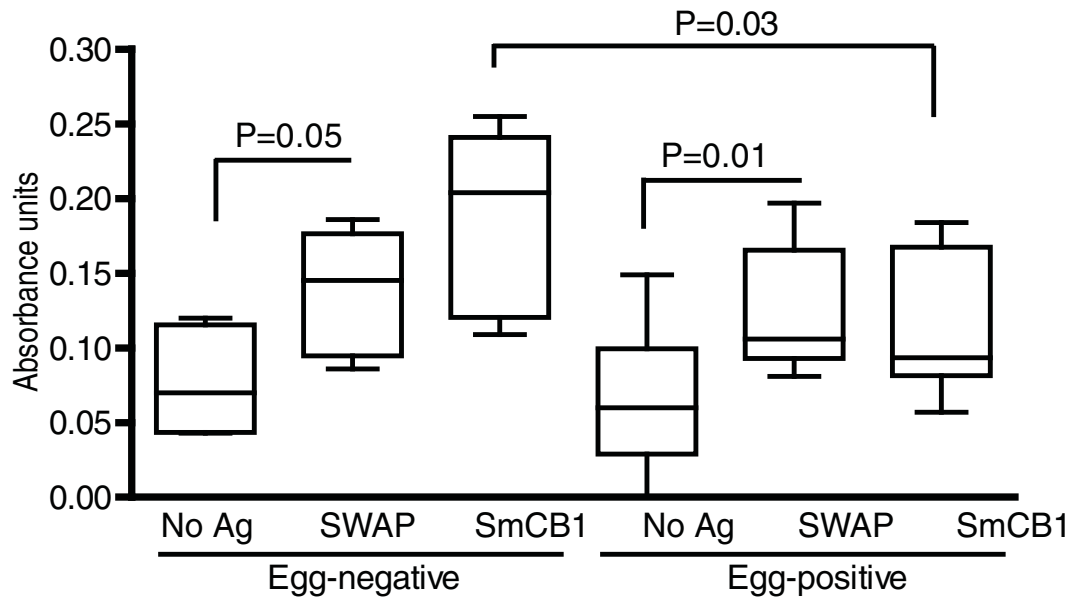

B

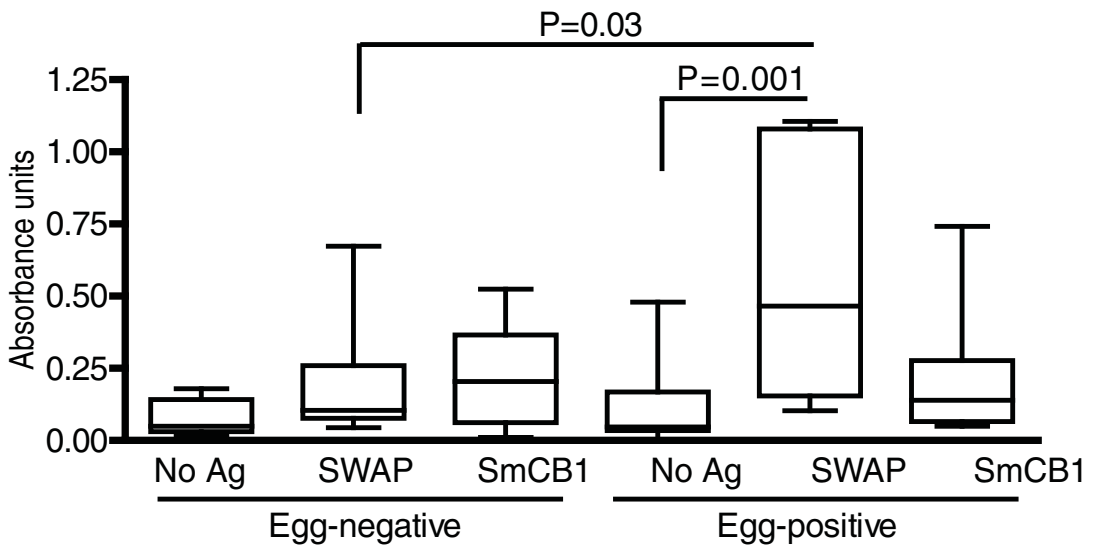

Figure 5 IgE responses to SmCB1 in exposed human subjects. Archived sera obtained from egg-positive and egg-negative (i.e. putatively resistant) subjects at the time of enrollment were assayed for $\operatorname{lgE}(\mathrm{A})$ and $\lg G 4$ responses (B) to SWAP and SmCB1 by ELISA. Boxes show the interquartile range and the median values obtained. Whiskers indicate the ranges of the data. $P$ values were calculated by Dunn's post-test following Kruskal-Wallis test. Number of individuals in each group: egg-positive $(n=13)$ egg-negative $(n=8)$.

orthomyxoviruses [45] and hepadnaviruses [46], responses to immunodominant epitopes can select for escape mutants that avoid immune killing, resulting in viral persistence and transmission to new hosts. In more complex eukaryotic pathogens such as Trypanosoma, narrowly focused responses to immunodominant antigens, such as the variant surface glycoproteins of African trypanosomes [47] and members of the highly diverse trans-sialidase gene family of T. cruzi [48], provide the parasites with a mechanism for immune evasion through antigenic variation. In Toxoplasma gondii, extreme focusing of the immune response on certain immunodominant members of the SRS (SAG1related sequences) family of surface antigens may promote parasite survival by distracting the host response away from other epitopes [49]. Focused immune responses to immunodominant antigens can therefore confer survival and transmission advantages, resulting in selection for and conservation of immunodominant antigens in a wide variety of unrelated pathogens. It is tempting to speculate that SmCB1 serves a similar function for schistosomes by inducing responses that are advantageous to the parasite, perhaps by stimulating $\mathrm{CD}^{+} \mathrm{T}$ cell responses that provide essential signals for parasite development $[4,50]$. The possibility that secreted parasite proteases may have functions that extend beyond their nutritive role warrants further investigation. Indeed, schistosomes are known to release catalytically inactive forms of proteases [51,52], with amino acid sequences that are almost identical to the active forms, suggesting these molecules have functions distinct from host protein degradation.

While schistosome eggs are thought to be the major stimulus for Th2 response induction during schistosome 
infection [6], we recently demonstrated that schistosome worms induce type 2 responses before oviposition begins [14]. By showing here that in vivo ablation of cognate $\mathrm{T}$ cell help or blockade of IL-4 signaling both prevented the pre-patent SmCB1-specific IgE response, our data suggest that the pre-patent Th2 response to worms is functional and provides help for humoral responses to antigens such as $\mathrm{SmCB} 1$. Interestingly, Th2 response induction by prepatent schistosome infection has been documented previously. Exposure of mice and humans to cercariae of the avian schistosome Trichobilharzia regenti, which does not result in patent infection, also results in induction of $\operatorname{IgE}$ responses and sensitization of basophils to produce IL-4 [53]. Whether pre-patent Th2 responses influence the development of subsequent Th2 responses to schistosome eggs remains to be tested, but the hypothesis that prepatent infection primes the subsequent anti-egg response has been proposed by others [54].

Our analysis of sera from human subjects exposed to schistosome infection demonstrates that SmCB1 is also the target of an IgE response in humans. That putatively resistant individuals exhibit significantly higher levels of SmCB1-specific IgE than infected subjects agrees with various other data that implicate parasitespecific IgE in mediating protective functions upon exposure to schistosome infection [31,55]. For example, high levels of specific IgE correlate with acquisition of resistance to re-infection in humans [56] and specific IgE is suspected to mediate parasite killing in some laboratory animal models of schistosome infection [57]. However, resistance in well-defined cohorts of putatively resistant Brazilian subjects is thought to be mediated by Th1 responses to worm antigens [58], similar to the immunity induced in mice by exposure to irradiated cercariae [59]. An alternative explanation for our finding is that persistent IL-10 production in chronically infected individuals leads to diminished IgE production and elevated IgG4 titers [60]. In this case, high SmCB1-specific IgE levels in putatively resistant subjects could be explained by the absence of chronic infection in these individuals. On the other hand, there is evidence that responses to certain antigens, such as the S. mansoni tetraspanins TSP-1 and TSP-2, may contribute to resistance in putatively resistant subjects [61], so the role of SmCB1 as a protective antigen may warrant further investigation. It is plausible that a protease with significant nutritive function that is expressed from the very beginning of infection may be a target of protective immune responses. Immune responses to another worm protease, calpain, can mediate significant resistance to challenge infection [62] suggesting that proteases are viable targets for future vaccines.

\section{Conclusions}

In conclusion, our data suggest that SmCB1 is an immunodominant target of the immune response during pre-patent schistosome infection and that the response to this cysteine protease exhibits hallmarks of a Th2 response. These findings suggest that, in addition to the nutritive function associated with its proteolytic activity, SmCB1 may serve in an immunological capacity to induce responses that influence the outcome of schistosome infection. Modulation of immune responses to proteases might therefore impede the establishment of schistosome infections and represent a novel approach to the treatment and/or prophylaxis of schistosomiasis.

\section{Methods}

\section{Animals and parasites}

Wild type C57BL/6 and C57BL/6 $\mathrm{MHC} \mathrm{II}^{-1-}$ mice were purchased from National Cancer Institute (Frederick, MD) and Taconic (Hudson, NY) respectively, and maintained in a specific pathogen-free environment. Mixed male and female cercariae of Schistosoma mansoni (Puerto Rican strain) were obtained from infected Biomphalaria glabrata snails provided by Dr. Fred Lewis (BRI, Rockville, MD). To obtain separate male and female cercariae, individual B. glabrata snails were exposed to single miracidia and tested for cercarial production 4-6 weeks later. Mice were infected by immersion of the tail for $40 \mathrm{~min}$ in water containing 50-150 S. mansoni cercariae and were sacrificed 1-8 weeks later, depending on experiment. For plasma isolation, blood was obtained by cardiac puncture at euthanasia, collected into heparinized tubes and centrifuged at 3,300 $\times$ $g$ to remove cells. In IL-4 neutralization experiments, mice were treated twice weekly with $1 \mathrm{mg}$ of the neutralizing anti-mouse IL-4 antibody 11B11 administered by intraperitoneal injection, while control groups received control rat IgG or PBS. For preparation of schistosome worm antigen (SWAP), adult S. mansoni were perfused from the portal veins of infected mice and homogenized in PBS on ice. Insoluble material was removed by centrifugation at $16,100 \times g$ for $30 \mathrm{~min}$ at $4^{\circ} \mathrm{C}$ and the resulting supernatant stored at $-80^{\circ} \mathrm{C}$ after filter sterilization and determination of protein concentration by Bradford assay. In all experiments, experimental groups of mice were exposed at the same time to parasites from the same cercarial pool. All studies involving animals were performed in accordance with protocols approved by the USUHS Institutional Animal Care and Use Committee and included 5-10 mice in each group.

\section{ELISA quantification of mouse immunoglobulin isotypes}

For detection of SWAP- and SmCB1-specific IgM, IgG1, IgG2b and IgA, 4HBX plates (Immulon Thermo, MA) 
were coated with SWAP or recombinant SmCB1 antigen $(5 \mu \mathrm{g} / \mathrm{ml})$ in borate buffered saline (BBS) for 2 hours at room temperature (RT). After 5 washes and blocking with BBS containing $1 \%$ of fetal calf serum (FCS), the immune plasma were diluted in BBS (1:4, the optimal dilution resulting in the highest signal-to-noise ratio determined for each isotype beforehand) containing 0.02\% Tween 20 (BBST; Sigma, St Louis, MO) and applied to the plates for 2 hours at RT. For isotypes other than IgE, the plates were incubated with alkaline phosphatase-conjugated goat antibodies against mouse IgM, IgG1, IgG2b, or IgA (Southern Biotechnology Associates) diluted 1:1000 in BBST for 30 minutes at $\mathrm{RT}$ and then washed 10 times. The reaction was developed by addition of 4- methylumbelliferyl phosphate substrate (4-MUP; Sigma). Fluorescence was detected on a SPECTRAmax M2 microplate fluorometer (Molecular Devices, Sunnyvale, CA) at excitation and emission wavelengths of 360nm and $449 \mathrm{~nm}$, respectively. Detection of SmCB1-specific IgE was the same as for other isotypes, except that mice were infected specifically for IgE investigations and IgG was first adsorbed by incubating plasma samples with GammaBind G Sepharose (Amersham Biosciences, Uppsala, Sweden) overnight at $4^{\circ} \mathrm{C}$ prior to application to the assay plates. After washes and incubation with alkaline phosphatase-conjugated goat anti-mouse IgE (BD Biosciences, San Diego, CA), the reaction was developed by addition of $p$-nitrophenyl phosphate disodium salt substrate (PNPP; Pierce, Rockford, IL), stopped with $2 \mathrm{~N} \mathrm{NaOH}$ and absorbance measured at $405 \mathrm{~nm}$. Concentrations of total IgE were determined using a sandwich ELISA kit (Antibody Set for mouse IgE; BD Biosciences), according to the manufacturer's instructions.

\section{Immunoblotting}

Preparations of SWAP (15 $\mu \mathrm{g} /$ lane) were separated by SDS-PAGE on $12 \%$ Bis-Tris gels under reducing conditions, transferred to PVDF membranes and probed with plasma from infected mice at 1:500 dilution. Bound antibodies were detected using alkaline phosphatase-conjugated goat antibodies against mouse IgM, IgG1, IgG2b and IgA (Southern Biotechnology Associates, Birmingham, AL) diluted 1:1000, in conjunction with a chemiluminescent immunodetection system (WesternBreeze; Invitrogen, Carlsbad, CA).

\section{Recombinant S. mansoni cathepsin B1 (SmB1)}

The original DNA plasmid construct used to produce a recombinant pro-form of the $S$. mansoni cathepsin B1 (SmCB1, a.k.a. Sm31) cysteine protease in Pichia pastoris [63] was subjected to PCR-based site-directed mutagenesis in order to remove a putative glycosylation site. Specifically, threonine residues at positions 185 and
300 (see Genbank accession number AJ506157) were substituted for alanines. Otherwise, expression of the protein was as previously described [63]. Yeastexpressed SmCB1 was lyophilized in sodium phosphate buffer $0.05 \mathrm{M}, \mathrm{pH} 6.0$ for storage and solubilized at a concentration of $2.4 \mathrm{mg} / \mathrm{ml}$ prior to use.

\section{Human subjects}

Sera from human subjects residing in endemic areas near Governador Valadares, Minas Gerais State, in southeast Brazil, were analyzed anonymously for antibody isotype responses to SWAP and SmCB1. Archived sera were randomly selected from two groups of subjects characterized as either egg-positive or egg-negative according to semi-annual feces examination from 1997 to the present. Egg-positive subjects were defined as those that have tested positive for the presence of $S$. mansoni eggs in their feces on at least one occasion since monitoring began in 1997. All patients in this group are treated with praziquantel when a schistosome infection is detected, and some individuals have experienced multiple episodes of re-infection and treatment. Egg-negative ("endemic normal") subjects were defined as individuals that reside in areas of known transmission but who have (i) never tested positive for schistosome eggs on fecal examination since monitoring began in 1997 and (ii) have no known history of schistosome infection or praziquantel treatment. Antibody responses to SWAP and SmCB1 at the time of enrollment in $1997 / 1998$ were assessed by ELISA analysis of archived sera as described above for murine immunoglobulins, except that alkaline phosphatase-conjugated mouse antihuman IgG4 (BD Pharmingen, BD Biosciences, San Diego, CA) and goat anti-human IgE (Biosource, Camarillo, CA) were used as secondary reagents. Assays were developed by addition of PNPP, stopped with $2 \mathrm{~N}$ $\mathrm{NaOH}$ and absorbance measured at $405 \mathrm{~nm}$. All studies involving human subjects were conducted under the auspices of UNIVALE and were approved by the relevant ethics committees (UNIVALE, protocol PQ 015/ 07-4, approved 04 December, 2007; CONEP (Brasilia), Registration: 14004, protocol number 25000.078835/ 2007-81, approved 12 July, 2007). Written informed consent was obtained from all participating subjects and is maintained on file at UNIVALE.

\section{Statistical analyses}

Because unequal variances between experimental groups were frequently encountered in some experiments, stringent non-parametric tests were used throughout to test the significance of differences between experimental groups. For comparison of two groups, significance was tested using Mann-Whitney tests, and for experiments involving three groups or 
more, the significance of differences was tested using Kruskal-Wallis tests followed by Dunn's multiple comparison tests. Statistical analyses were performed with GraphPad Prism Version 4.0 software (GraphPad Software, Inc., San Diego, CA). P values of less than 0.05 were considered significant. Data are expressed as mean value \pm SEM. All data are representative of at least two independent experiments.

\section{Acknowledgements}

We thank Maria de Fátima da Silva, Marlucy Lima, Lilia Pires and Ivanete Santos for invaluable assistance with field studies and Sean Maynard for excellent technical assistance. S. mansoni-infected and non-infected $B$. glabrata snails were provided by Dr. Fred Lewis through National Institutes of Health/National Institute of Allergy and Infectious Diseases Contract N01 Al30026. Financial support was provided by NIH/NIAID grant R01 Al066227 (to SJD) and by CNPq fellowship 210320/2006-0 (to LAOF).

\section{Author details}

'Department of Microbiology and Immunology, Uniformed Services University of the Health Sciences, Bethesda, MD 20814, USA. ${ }^{2}$ Universidade Vale do Rio Doce (UNIVALE), Governador Valadares, Minas Gerais, Brazil. ${ }^{3}$ Fundação Nacional de Saúde - FUNASA/BH, Belo Horizonte, Minas Gerais, Brazil. ${ }^{4}$ Sandler Center for Basic Research in Parasitic Diseases, California Institute for Quantitative Biosciences (QB3), University of California, San Francisco, 1700 4th St., San Francisco, CA 94158, USA. ${ }^{5} \mathrm{NIH} / \mathrm{NIAID} / \mathrm{LPD}, 4$ Center Drive, Building 4, Room B1-06, Bethesda, MD 20892, USA. ${ }^{6} 1471$ Hopkins Street, Berkeley, CA 94702, USA. 'eiden Malaria Research Group, Leiden University Medical Centre, afd. Parasitologie, Albinusdreef 2, Kamer P4-35, 2333 ZA Leiden, Netherlands.

\section{Authors' contributions}

LAOF participated in the design and coordination of the study, performed the experimental work and drafted the manuscript. EWL participated in the design and coordination of the study and assisted with assay performance and data collection. ECM performed the epidemiological analyses. MC provided parasite materials and performed biochemical assays. JD developed methodologies for the expression and purification of recombinant parasite proteases. MD established expression systems for recombinant parasite proteases, developed purification methodologies and performed biochemical analyses. MS contributed expertise to the development of recombinant protease expression and purification systems and provided expert advice. CRC participated in the design of the study and supplied essential reagents and advice. SJD conceived, designed and coordinated the study, participated in assay performance and data collection and drafted the manuscript. All authors read and approved the manuscript.

Received: 26 January 2010 Accepted: 15 November 2010 Published: 15 November 2010

\section{References}

1. Pearce EJ, Sher A: Mechanisms of immune evasion in schistosomiasis. Contrib Microbiol Immunol 1987, 8:219-232.

2. McManus DP, Loukas A: Current status of vaccines for schistosomiasis. Clin Microbiol Rev 2008, 21:225-242.

3. Hewitson JP, Hamblin PA, Mountford AP: Immunity induced by the radiation-attenuated schistosome vaccine. Parasite Immunol 2005, 27:271-280.

4. Davies SJ, Grogan JL, Blank RB, Lim KC, Locksley RM, McKerrow JH: Modulation of Blood Fluke Development in the Liver by Hepatic CD4+ Lymphocytes. Science 2001, 294:1358-1361.

5. Harrison RA, Doenhoff MJ: Retarded development of Schistosoma mansoni in immunosuppressed mice. Parasitology 1983, 86:429-438.

6. Pearce EJ: Priming of the immune response by schistosome eggs. Parasite Immunol 2005, 27:265-270.

7. Grzych JM, Pearce E, Cheever A, Caulada ZA, Caspar P, Heiny S, Lewis F, Sher A: Egg deposition is the major stimulus for the production of Th2 cytokines in murine schistosomiasis mansoni. J Immunol 1991, 146:1322-1327.

8. Stadecker MJ, Hernandez HJ, Asahi H: The identification and characterization of new immunogenic egg components: implications for evaluation and control of the immunopathogenic T cell response in schistosomiasis. Mem Inst Oswaldo Cruz 2001, 96(Suppl):29-33.

9. Chen $Y$, Boros DL: Identification of the immunodominant T cell epitope of p38, a major egg antigen, and characterization of the epitope-specific Th responsiveness during murine schistosomiasis mansoni. J Immunol 1998, 160:5420-5427.

10. Hernandez HJ, Edson CM, Harn DA, lanelli CJ, Stadecker MJ: Schistosoma mansoni: genetic restriction and cytokine profile of the CD4 + T helper cell response to dominant epitope peptide of major egg antigen Smp40. Exp Parasitol 1998, 90:122-130.

11. Everts B, Perona-Wright G, Smits HH, Hokke CH, van der Ham AJ, Fitzsimmons CM, Doenhoff MJ, van der Bosch J, Mohrs K, Haas H, et al: Omega-1, a glycoprotein secreted by Schistosoma mansoni eggs, drives Th2 responses. J Exp Med 2009, 206:1673-1680.

12. Steinfelder S, Andersen JF, Cannons JL, Feng CG, Joshi M, Dwyer D, Caspar P, Schwartzberg PL, Sher A, Jankovic D: The major component in schistosome eggs responsible for conditioning dendritic cells for Th2 polarization is a T2 ribonuclease (omega-1). J Exp Med 2009, 206:1681-1690.

13. Pearce EJ, Caspar P, Grzych JM, Lewis FA, Sher A: Downregulation of Th1 cytokine production accompanies induction of Th2 responses by a parasitic helminth, Schistosoma mansoni. J Exp Med 1991, 173:159-166.

14. de Oliveira Fraga LA, Torrero MN, Tocheva AS, Mitre E, Davies SJ: Induction of type 2 responses by schistosome worms during prepatent infection. J Infect Dis 2010, 201:464-472.

15. Balloul JM, Sondermeyer P, Dreyer D, Capron M, Grzych JM, Pierce RJ, Carvallo D, Lecocq JP, Capron A: Molecular cloning of a protective antigen of schistosomes. Nature 1987, 326:149-153.

16. Pearce EJ, James SL, Dalton J, Barrall A, Ramos C, Strand M, Sher A: Immunochemical characterization and purification of Sm-97, a Schistosoma mansoni antigen monospecifically recognized by antibodies from mice protectively immunized with a nonliving vaccine. J Immunol 1986, 137:3593-3600.

17. Strand M, Dalton JP, Tom TD: Characterization and cloning of Schistosoma mansoni immunogens recognized by protective antibodies. Acta Trop Supp/ 1987, 12:75-82.

18. Correa-Oliveira R, Pearce EJ, Oliveira GC, Golgher DB, Katz N, Bahia LG, Carvalho OS, Gazzinelli G, Sher A: The human immune response to defined immunogens of Schistosoma mansoni: elevated antibody levels to paramyosin in stool-negative individuals from two endemic areas in Brazil. Trans R Soc Trop Med Hyg 1989, 83:798-804.

19. El Ridi R, Farouk F, Sherif M, Al-Sherbiny M, Osman A, El Gengehi N, Shoemaker CB: $T$ and $B$ cell reactivity to a $42-k D a$ protein is associated with human resistance to both schistosomiasis mansoni and haematobium. J Infect Dis 1998, 177:1364-1372.

20. Goudot-Crozel V, Caillol D, Djabali M, Dessein AJ: The major parasite surface antigen associated with human resistance to schistosomiasis is a 37-kD glyceraldehyde-3P-dehydrogenase. J Exp Med 1989, 170:2065-2080.

21. Kearney ER, Pape KA, Loh DY, Jenkins MK: Visualization of peptide-specific T cell immunity and peripheral tolerance induction in vivo. Immunity 1994, 1:327-339.

22. Kawabe T, Naka T, Yoshida K, Tanaka T, Fujiwara H, Suematsu S, Yoshida N, Kishimoto T, Kikutani H: The immune responses in CD40-deficient mice: impaired immunoglobulin class switching and germinal center formation. Immunity 1994, 1:167-178.

23. Caffrey CR, McKerrow JH, Salter JP, Sajid M: Blood ' $n$ ' guts: an update on schistosome digestive peptidases. Trends Parasitol 2004, 20:241-248.

24. Chappell CL, Hackel J, Davis AH: Cloned Schistosoma mansoni proteinase (hemoglobinase) as a putative serodiagnostic reagent. J Clin Microbiol 1989, 27:196-198.

25. Grogan J, Rotmans P, Ghoneim H, Deelder A, Yazdanbakhsh M, Klinkert MQ: Recognition of Schistosoma mansoni cathepsins B and $L$ by human $\lg G 1$ and IgG4 antibodies. Parasite Immunol 1997, 19:215-220.

26. Klinkert MQ, Bommert K, Moser D, Felleisen R, Link G, Doumbo O, Beck E: Immunological analysis of cloned Schistosoma mansoni antigens Sm31 and Sm32 with sera of schistosomiasis patients. Trop Med Parasitol 1991, 42:319-324. 
27. Chappell $\mathrm{CL}$, Dresden $\mathrm{MH}$ : Antibody response to a purified parasite proteinase (SMw32) in Schistosoma mansoni infected mice. Am J Trop Med Hyg 1988, 39:66-73.

28. Vercelli D, Jabara HH, Arai K, Geha RS: Induction of human IgE synthesis requires interleukin 4 and $T / B$ cell interactions involving the $T$ cell receptor/CD3 complex and MHC class II antigens. J Exp Med 1989, 169:1295-1307.

29. LoVerde PT, Chen L: Schistosome female reproductive development. Parasitology Today 1991, 7:303-307.

30. McCoy KD, Harris NL, Diener P, Hatak S, Odermatt B, Hangartner L, Senn BM, Marsland BJ, Geuking MB, Hengartner $H$, et al: Natural IgE production in the absence of MHC Class II cognate help. Immunity 2006, 24:329-339.

31. Hagan P, Blumenthal UJ, Dunn D, Simpson AJ, Wilkins HA: Human IgE, $\operatorname{lgG} 4$ and resistance to reinfection with Schistosoma haematobium. Nature 1991, 349:243-245.

32. Berriman M, Haas BJ, LoVerde PT, Wilson RA, Dillon GP, Cerqueira GC, Mashiyama ST, Al-Lazikani B, Andrade LF, Ashton PD, et al: The genome of the blood fluke Schistosoma mansoni. Nature 2009, 460:352-358.

33. Jolly ER, Chin CS, Miller S, Bahgat MM, Lim KC, DeRisi J, McKerrow JH: Gene expression patterns during adaptation of a helminth parasite to different environmental niches. Genome Biol 2007, 8:R65.

34. Mikhail MM, Mansour MM, Farid Z, Harrison R: Schistosoma mansoni antigens applicable to diagnosis of prepatent infections. J Egypt SoC Parasitol 1997, 27:1-20

35. Klinkert MQ, Felleisen R, Link G, Ruppel A, Beck E: Primary structures of Sm31/32 diagnostic proteins of Schistosoma mansoni and their identification as proteases. Mol Biochem Parasitol 1989, 33:113-122.

36. Skelly PJ, Shoemaker CB: Schistosoma mansoni proteases Sm31 (cathepsin B) and Sm32 (legumain) are expressed in the cecum and protonephridia of cercariae. J Parasitol 2001, 87:1218-1221.

37. Štefanić S, Dvořák J, Horn M, Braschi S, Sojka D, Ruelas D, Suzuki B, Lim KC, Hopkins SD, McKerrow JH, Caffrey CR: RNA interference in Schistosoma mansoni schistosomula: selectivity, sensitivity and operation for largerscale screening. PLOS Negl Trop Dis 2010.

38. Correnti JM, Brindley PJ, Pearce EJ: Long-term suppression of cathepsin B levels by RNA interference retards schistosome growth. Mol Biochem Parasitol 2005, 143:209-215.

39. Abdulla MH, Lim KC, Sajid M, McKerrow JH, Caffrey CR: Schistosomiasis mansoni: novel chemotherapy using a cysteine protease inhibitor. PLoS Med 2007, 4:e14

40. Medzhitov R: Origin and physiological roles of inflammation. Nature 2008, 454:428-435.

41. Sokol CL, Barton GM, Farr AG, Medzhitov R: A mechanism for the initiation of allergen-induced T helper type 2 responses. Nat Immunol 2008, 9:310-318.

42. Lecaille F, Kaleta J, Bromme D: Human and parasitic papain-like cysteine proteases: their role in physiology and pathology and recent developments in inhibitor design. Chem Rev 2002, 102:4459-4488.

43. McKerrow JH: Cysteine proteases of parasites: A remarkable diversity of function. Perspectives in Drug Discovery and Design 1994, 2:437-444.

44. McMichael AJ, Phillips RE: Escape of human immunodeficiency virus from immune control. Annu Rev Immunol 1997, 15:271-296.

45. Suzuki Y: Natural selection on the influenza virus genome. Mol Biol Evol 2006, 23:1902-1911.

46. Cooreman MP, Leroux-Roels G, Paulij WP: Vaccine- and hepatitis B immune globulin-induced escape mutations of hepatitis $B$ virus surface antigen. J Biomed Sci 2001, 8:237-247.

47. Sternberg JM: Human African trypanosomiasis: clinical presentation and immune response. Parasite Immunol 2004, 26:469-476.

48. Martin DL, Weatherly DB, Laucella SA, Cabinian MA, Crim MT, Sullivan S, Heiges M, Craven SH, Rosenberg CS, Collins MH, et al: CD8+ T-Cell responses to Trypanosoma cruzi are highly focused on strain-variant trans-sialidase epitopes. PLOS Pathog 2006, 2:e77.

49. Jung C, Lee CY, Grigg ME: The SRS superfamily of Toxoplasma surface proteins. Int J Parasitol 2004, 34:285-296.

50. Lamb EW, Walls CD, Pesce JT, Riner DK, Maynard SK, Crow ET, Wynn TA, Schaefer BC, Davies SJ: Blood fluke exploitation of non-cognate CD4+ T cell help to facilitate parasite development. PLOS Pathog 2010, 6: e1000892.
51. Delcroix M, Medzihradsky K, Caffrey CR, Fetter RD, McKerrow JH: Proteomic analysis of adult S. mansoni gut contents. Mol Biochem Parasitol 2007, 154:95-97.

52. Merckelbach A, Hasse S, Dell R, Eschlbeck A, Ruppel A: cDNA sequences of Schistosoma japonicum coding for two cathepsin B-like proteins and Sj32. Trop Med Parasitol 1994, 45:193-198.

53. Lichtenbergova L, Kolbekova $P$, Kourilova $P$, Kasny M, Mikes L, Haas $H$, Schramm G, Horak P, Kolarova L, Mountford AP: Antibody responses induced by Trichobilharzia regenti antigens in murine and human hosts exhibiting cercarial dermatitis. Parasite Immunol 2008, 30:585-595.

54. Leptak CL, McKerrow JH: Schistosome egg granulomas and hepatic expression of TNF-alpha are dependent on immune priming during parasite maturation. J Immunol 1997, 158:301-307.

55. Rihet $P$, Demeure $C E$, Bourgois A, Prata A, Dessein AJ: Evidence for an association between human resistance to Schistosoma mansoni and high anti-larval IgE levels. Eur J Immunol 1991, 21:2679-2686.

56. Ganley-Leal LM, Mwinzi PN, Cetre-Sossah CB, Andove J, Hightower AW, Karanja DM, Colley DG, Secor WE: Correlation between eosinophils and protection against reinfection with Schistosoma mansoni and the effect of human immunodeficiency virus type 1 coinfection in humans. Infect Immun 2006, 74:2169-2176.

57. Nyindo M, Kariuki TM, Mola PW, Farah IO, Elson L, Blanton RE, King CL: Role of adult worm antigen-specific immunoglobulin $E$ in acquired immunity to Schistosoma mansoni infection in baboons. Infect Immun 1999, 67:636-642.

58. Correa-Oliveira R, Caldas IR, Gazzinelli G: Natural versus drug-induced resistance in Schistosoma mansoni infection. Parasitol Today 2000, 16:397-399.

59. Wynn TA, Hoffmann KF: Defining a schistosomiasis vaccination strategy is it really Th1 versus Th2? Parasitol Today 2000, 16:497-501.

60. Maizels RM, Balic A, Gomez-Escobar N, Nair M, Taylor MD, Allen JE: Helminth parasites-masters of regulation. Immunol Rev 2004, 201:89-116.

61. Tran MH, Pearson MS, Bethony JM, Smyth DJ, Jones MK, Duke M, Don TA, McManus DP, Correa-Oliveira R, Loukas A: Tetraspanins on the surface of Schistosoma mansoni are protective antigens against schistosomiasis. Nat Med 2006, 12:835-840.

62. Jankovic D, Aslund L, Oswald IP, Caspar P, Champion C, Pearce E, Coligan JE, Strand M, Sher A, James SL: Calpain is the target antigen of a Th1 clone that transfers protective immunity against Schistosoma mansoni. J Immunol 1996, 157:806-814

63. Sajid M, McKerrow JH, Hansell E, Mathieu MA, Lucas KD, Hsieh I, Greenbaum D, Bogyo M, Salter JP, Lim KC, et al: Functional expression and characterization of Schistosoma mansoni cathepsin B and its transactivation by an endogenous asparaginyl endopeptidase. Mol Biochem Parasitol 2003, 131:65-75.

doi:10.1186/1471-2172-11-56

Cite this article as: de Oliveira Fraga et al.: Rapid induction of $\operatorname{lgE}$ responses to a worm cysteine protease during murine pre-patent schistosome infection. BMC Immunology 2010 11:56.

\section{Submit your next manuscript to BioMed Central and take full advantage of:}

- Convenient online submission

- Thorough peer review

- No space constraints or color figure charges

- Immediate publication on acceptance

- Inclusion in PubMed, CAS, Scopus and Google Scholar

- Research which is freely available for redistribution

Submit your manuscript at www.biomedcentral.com/submit
Biomed Central 